\title{
Densities and filling factors of the diffuse ionized gas in the Solar neighbourhood
}

\author{
E. M. Berkhuijsen and P. Müller
}

\author{
Max-Planck-Institut für Radioastronomie, Auf dem Hügel 69, 53121 Bonn, Germany \\ e-mail: [eberkhuijsen;peter] @mpifr-bonn.mpg.de
}

Received 28 February 2008 / Accepted 13 July 2008

\begin{abstract}
Aims. We analyse electron densities and filling factors of the diffuse ionized gas (DIG) in the Solar neighbourhood. Methods. We have combined dispersion measures and emission measures towards 38 pulsars at distances known to better than $50 \%$, from which we derived the mean density in clouds, $N_{\mathrm{c}}$, and their volume filling factor, $F_{\mathrm{v}}$, averaged along the line of sight. The emission measures were corrected for absorption by dust and contributions from beyond the pulsar distance.

Results. The scale height of the electron layer for our sample is $0.93 \pm 0.13 \mathrm{kpc}$ and the midplane electron density is $0.023 \pm 0.004 \mathrm{~cm}^{-3}$, in agreement with earlier results. The average density along the line of sight is $\left\langle n_{\mathrm{e}}\right\rangle=0.018 \pm 0.002 \mathrm{~cm}^{-3}$ and is nearly constant. Since $\left\langle n_{\mathrm{e}}\right\rangle=F_{\mathrm{v}} N_{\mathrm{c}}$, an inverse relationship between $F_{\mathrm{v}}$ and $N_{\mathrm{c}}$ is expected. We find $F_{\mathrm{v}}\left(N_{\mathrm{c}}\right)=(0.011 \pm 0.003) N_{\mathrm{c}}^{-1.20 \pm 0.13}$, which holds for the ranges $N_{\mathrm{c}}=0.05-1 \mathrm{~cm}^{-3}$ and $F_{\mathrm{v}}=0.4-0.01$. Near the Galactic plane the dependence of $F_{\mathrm{v}}$ on $N_{\mathrm{c}}$ is significantly stronger than away from the plane. $F_{\mathrm{v}}$ does not systematically change along or perpendicular to the Galactic plane, but the spread about the mean value of $0.08 \pm 0.02$ is considerable. The total pathlength through the ionized regions increases linearly to about $80 \mathrm{pc}$ towards $|z|=1 \mathrm{kpc}$. Conclusions. Our study of $F_{\mathrm{v}}$ and $N_{\mathrm{c}}$ of the DIG is the first one based on a sample of pulsars with known distances. We confirm the existence of a tight, nearly inverse correlation between $F_{\mathrm{v}}$ and $N_{\mathrm{c}}$ in the DIG. The exact form of this relation depends on the regions in the Galaxy probed by the pulsar sample. The inverse $F_{\mathrm{v}}-N_{\mathrm{c}}$ relation is consistent with a hierarchical, fractal density distribution in the DIG caused by turbulence. The observed near constancy of $\left\langle n_{\mathrm{e}}\right\rangle$ then is a signature of fractal structure in the ionized medium, which is most pronounced outside the thin disk.
\end{abstract}

Key words. Galaxy: disk - H II regions - ISM: clouds - ISM: structure

\section{Introduction}

The full-sky $\mathrm{H} \alpha$ maps of the Milky Way that have recently become available (Dickinson et al. 2003; Finkbeiner 2003; Bennett et al. 2003; Hinshaw et al. 2007) show a spectacular variation in intensity and structure. The classical H II regions in the disc are surrounded by extended, diffuse ionized gas (DIG) visible up to high latitudes, and supernova explosions, shocks and turbulence in the interstellar medium (ISM) have produced a wealth of cloud shapes, filaments, shells and voids.

Madsen et al. (2006) found the variations in structure to be accompanied by variations in electron temperature. They derived a mean temprature of the DIG of about $8000 \mathrm{~K}$ with strong differences between lines of sight.

A number of authors derived a scale height of the DIG in the Solar neighbourhood of about $1 \mathrm{kpc}$ from the increase of the dispersion measures of pulsars with increasing distance to the Galactic plane (Reynolds 1991b; Bhattacharia \& Verbunt 1991; Nordgren et al. 1992; Gomez et al. 2001; Cordes \& Lazio 2003). Using dispersion measures towards 4 pulsars in globular clusters, Reynolds (1991a) obtained a filling fraction of the DIG of $\$ 0.2$ through the full layer and a mean density in clouds of about $0.08 \mathrm{~cm}^{-3}$. This was recently confirmed by Berkhuijsen et al. (2006) from a much larger pulsar sample. These authors also found an increase of the filling factor with distance from the plane. Already the scanty data that were available to Kulkarni \& Heiles (1988) had indicated such an increase.

Turbulence causing hierarchical, fractal structure leads to an inverse relationship between the mean filling factor along the line of sight, $F_{\mathrm{v}}$, and the mean density in ionized regions, $N_{\mathrm{c}}$ (Fleck 1996; Elmegreen 1998, 1999). Pynzar (1993), who was the first to investigate this relationship, derived $F_{\mathrm{v}} \propto N_{\mathrm{c}}^{-0.7}$ for a combination of DIG and H II regions, which was confirmed by Berkhuijsen (1998). In an extensive study, Berkhuijsen et al. (2006, hereafter BMM) obtained $F_{\mathrm{v}} \propto N_{\mathrm{c}}^{-1.07 \pm 0.03}$ for the DIG from a sample of 157 pulsars at latitudes $|b|>5^{\circ}$. They used dispersion measures from the catalogue of Hobbs \& Manchester (2003; see also Manchester et al. 2005), emission measures from the WHAM survey (Haffner et al. 2003), corrected for absorption (Diplas \& Savage 1994) as well as for contributions from beyond the pulsars, and pulsar distances from the model of the electron distribution in the Galaxy of Cordes \& Lazio (2002). The statistical error in pulsar distances $<3 \mathrm{kpc}$, where $75 \%$ of the pulsars in the sample are located, is about $25 \%$ which is much smaller than the intrinsic spread in the dispersion measures and emission measures. Therefore, random errors in the model distances will not have influenced the statistical results. However, the distances from the model may also contain a systematic error. Based on these distances, the radial distribution of pulsars has a maximum at $R=3.5 \mathrm{kpc}$ from the Galactic centre (Lorimer 2004; Yusifov \& Küçük 2004), whereas other population I objects (H2, H I, H II regions) peak near $R=5 \mathrm{kpc}$. The smoothness of the electron density model may lead to pulsar distances that are too large.

So it is important to check the results of BMM for pulsars with distances derived from observations. Since BMM did their work, the sample of pulsars with measured distances has increased considerably. Our new analysis is based on 38 pulsars, 
the distances of which are known to better than 50\%. A further decrease of the constraint on the distance errror would make the sample too small for a statistical analysis. We also used an improved absorption correction of the emission measure towards each pulsar instead of the statistical correction applied by BMM.

\section{Basics and data}

\subsection{Basic relations}

The expressions for dispersion measure, $D M$, and emission measure, $E M$, towards a pulsar at distance $D$ (in pc) can be written in various ways:

$D M=\int_{0}^{\mathrm{D}} n_{\mathrm{e}}(l) \mathrm{d} l=\left\langle n_{\mathrm{e}}\right\rangle D=N_{\mathrm{c}} F_{\mathrm{v}} D=N_{\mathrm{c}} L_{\mathrm{e}}$,
$E M=\int_{0}^{\mathrm{D}} n_{\mathrm{e}}^{2}(l) \mathrm{d} l=\left\langle n_{\mathrm{e}}^{2}\right\rangle D=N_{\mathrm{c}}^{2} F_{\mathrm{v}} D=N_{\mathrm{c}}^{2} L_{\mathrm{e}}$,

where $n_{\mathrm{e}}(l)$ (in $\mathrm{cm}^{-3}$ ) is the local electron density at point $l$ along the line of sight, $L_{\mathrm{e}}$ (in pc) the total pathlength through the regions containing free electrons (clouds, clumps) and $N_{\mathrm{c}}$ (in $\mathrm{cm}^{-3}$ ) the average density in these regions; between clouds the electron density is assumed to be negligible (see Fig. 1 in BMM). Furthermore, $\left\langle n_{\mathrm{e}}\right\rangle$ and $\left\langle n_{\mathrm{e}}^{2}\right\rangle$ are averages along $D$, and $F_{\mathrm{v}}=L_{\mathrm{e}} / D$ is the fraction of the line of sight in clouds, which approximates the volume filling factor $F_{\mathrm{v}}$ if there are several clouds along the line of sight (BMM). Note that the third equality in Eq. (2) is only valid when the average density of each cloud $n_{\mathrm{c}}$ along the line of sight is the same: then $\left\langle n_{\mathrm{e}}^{2}\right\rangle=\left\langle n_{\mathrm{c}}^{2}\right\rangle F_{\mathrm{v}}=N_{\mathrm{c}}^{2} F_{\mathrm{v}}$. Thus $N_{\mathrm{c}}$ and $F_{\mathrm{v}}$ are approximations of the true average density in clouds and their filling factor.

Combining Eqs. (1) ${ }^{1}$ and (2) we have

$N_{\mathrm{c}}=E M / D M$,

$F_{\mathrm{v}}=D M^{2} / E M D$.

The derived quantities $N_{\mathrm{c}}$ and $F_{\mathrm{v}}$ are connected by the simple relation $\left\langle n_{\mathrm{e}}\right\rangle=F_{\mathrm{v}} N_{\mathrm{c}}$ following from the third equality in Eq. (1). As the mean electron density in clouds will not be constant, our estimate of $N_{\mathrm{c}}$ will be too high because of the $n_{\mathrm{e}}^{2}$ dependence of $E M$. Recently, Hill et al. (2008) found that the ratio between the most probable density and $N_{\mathrm{c}}$ varies between 0.95 and 0.4 in the mildly supersonic cases of their MHD simulations (scaled to observations). Thus, our values of $N_{\mathrm{c}}$ may be too high by a factor $\$ 2$; the corresponding values of $F_{\mathrm{v}}$ will then be too low by the same factor.

\subsection{The data}

We collected a sample of 78 pulsars with distances known to better than $50 \%$ from the literature. This error is negligible compared to the large intrinsic spread in $D M$ (a factor of 2) and $E M$ (a factor of 4). The sample consists of 52 pulsars from the list of Gomez et al. (2001), where we used more recent distances when available, and 26 pulsars with parallactic distances taken from Hobbs et al. (2005, and references therein).

The dispersion measures came from the catalogue of Manchester et al. (2005). To find the emission measures in the pulsar directions we used the full-sky $\mathrm{H} \alpha$ map of Finkbeiner (2003) corrected for extinction as described by Dickinson et al. (2003), who argued that only one third of the dust in the line of

${ }_{1}$ Note that we write $F_{\mathrm{v}}$ and $N_{\mathrm{c}}$ where BMM used $\bar{f}_{\mathrm{v}}$ and $\bar{n}_{\mathrm{c}}$. sight effectively absorbs $\mathrm{H} \alpha$ emission. The extinction-corrected emission measure is

$E M_{\mathrm{c}}=E M \times 0.34 A \quad$ with $A=\exp (2.4 E(B-V))$,

where $E M$ is the observed emission measure and $A$ the correction recommended by Finkbeiner (2003). Corrections of more than 1 mag are uncertain and cannot be used. Therefore we had to remove 38 pulsars from the sample, most of which are at latitudes $|b|<5^{\circ}$. We also removed 2 pulsars with high $E M$ and DM (J0613-0200 and J1807+0943) indicating H II regions on their lines of sight. Thus for the analysis a sample of 38 pulsars is available, for 23 of which a parallactic distance is known. The errors in the distances to 28 pulsars are smaller than $20 \%$ $(\Delta D / D<0.2)$ and those in the distances to the other 10 pulsars are less than $50 \%(0.2<\Delta D / D<0.5)$. As 21 pulsars are located at $D<2 \mathrm{kpc}$, the sample is heavily weighted to the Solar neighbourhood; 13 pulsars are in globular clusters at distances $>2 \mathrm{kpc}$. The main parameters of the 38 pulsars in the sample are listed in Table 1, which also gives the references to the distances used. The sightlines to these pulsars probe the DIG.

In the direction of nearby pulsars and of pulsars near the Galactic plane a significant amount of the emission measure originates beyond the pulsar (see Fig. 5 in BMM). Reynolds (1997) and Haffner et al. (2003) have shown that the vertical electron distribution derived from $\mathrm{H} \alpha$ observations is fairly well described by an exponential. Then the emission measure up to the pulsar, $E M_{\mathrm{p}}$, is obtained from

$E M_{\mathrm{p}} \sin |b|(z)=E M_{\mathrm{c}} \sin |b|\left(1-\exp \left(-\left|Z_{\mathrm{p}}\right| / h_{\mathrm{e}}\right)\right)$

where $Z_{\mathrm{p}}$ is the distance of the pulsar to the Galactic plane and $h_{\mathrm{e}}$ is the scale height of $n_{\mathrm{e}}^{2}(z)$. Deviations from a smooth, planeparallel layer will cause scatter in $E M_{\mathrm{p}}$. The mean of $E M_{\mathrm{c}} \sin |b|$ for our sample is $2.8 \pm 0.3 \mathrm{~cm}^{-6} \mathrm{pc}$, which is the extinctioncorrected emission measure through the full layer. We derive the scale height $h_{\mathrm{e}}$ in the next section.

\section{Scale height of electrons}

We first estimated the scale height $h_{\mathrm{e}}$ of $n_{\mathrm{e}}^{2}(z)$ using Eqs. (12) and (13) of BMM, expecting that also for our sample the average filling factor $F_{\mathrm{v}}(z)$ would increase with $|z|$. This yielded $280 \mathrm{pc}<$ $h_{\mathrm{e}}<510 \mathrm{pc}$ with a best value of $390 \mathrm{pc}$, giving the observed maximum values of $D M \sin |b|=22 \pm 2 \mathrm{~cm}^{-3}$ pc (see Fig. 1) and $E M_{\mathrm{p}} \sin |b|=2.8 \pm 0.3 \mathrm{~cm}^{-6} \mathrm{pc}$. However, for this entire range of $h_{\mathrm{e}}, F_{\mathrm{v}}(z)$ appeared to be essentially constant for our sample (see Fig. 4d), thus also the local filling factor $f(z)$ is $\simeq$ const. As is easily seen, this indicates that the scale height of $n_{\mathrm{e}}^{2}(z)$ is about half that of $n_{\mathrm{e}}(z)$.

Following BMM, we describe the dispersion measure perpendicular to the Galactic plane as

$$
\begin{aligned}
D M \sin |b|(z) & =\int_{0}^{\left|Z_{\mathrm{p}}\right|} n_{\mathrm{e}}(z) \mathrm{d} z=\int_{0}^{\left|Z_{\mathrm{p}}\right|} f(z) n_{\mathrm{c}}(z) \mathrm{d} z \\
& =f_{0} n_{\mathrm{c} 0} h\left(1-\exp \left(-\left|Z_{\mathrm{p}}\right| / h\right)\right)
\end{aligned}
$$

where we assume that $f(z)=f_{0}$ and the local density in clouds $n_{\mathrm{c}}(z)$ is an exponential with midplane value $n_{\mathrm{c} 0}$ and scale height $h ; Z_{\mathrm{p}}$ is the distance of the pulsar to the midplane. 
Table 1. Parameters of the 38 pulsars in the final sample.

\begin{tabular}{|c|c|c|c|c|c|c|c|c|c|c|}
\hline $\begin{array}{c}\text { PULSAR } \\
\text { Jname }\end{array}$ & $\begin{array}{r}L O N G \\
{\left[{ }^{\circ}\right]} \\
\end{array}$ & $\begin{array}{r}L A T \\
{\left[{ }^{\circ}\right]}\end{array}$ & $\begin{array}{c}D M^{a} \\
{\left[\mathrm{~cm}^{-3} \mathrm{pc}\right]}\end{array}$ & $D$ & $\begin{array}{l}\Delta D^{b} \\
\mathrm{c}]\end{array}$ & REF & $\begin{array}{c}E M_{\mathrm{c}} \\
{\left[\mathrm{cm}^{-}\right.}\end{array}$ & $\begin{array}{l}E M_{\mathrm{p}} \\
\mathrm{pc}]\end{array}$ & $\begin{array}{c}N_{\mathrm{c}} \\
{\left[\mathrm{cm}^{-3}\right]}\end{array}$ & $F_{\mathrm{v}}$ \\
\hline J0437-4715 & 253.40 & -42.00 & 2.65 & 159 & 5 & 1 & 1.8 & 0.4 & 0.137 & 0.122 \\
\hline J0814+7429 & 140.00 & 31.62 & 6.12 & 433 & 8 & 5 & 1.9 & 0.7 & 0.116 & 0.122 \\
\hline J0826+2637 & 197.00 & 31.70 & 19.45 & 360 & 80 & 6 & 4.8 & 1.6 & 0.081 & 0.665 \\
\hline J0953+0755 & 228.91 & 43.70 & 2.96 & 262 & 5 & 5 & 3.2 & 1.0 & 0.351 & 0.032 \\
\hline $\mathrm{J} 1136+1551$ & 241.91 & 69.19 & 4.86 & 350 & 20 & 5 & 1.5 & 0.8 & 0.155 & 0.090 \\
\hline $\mathrm{J} 1239+2453$ & 252.45 & 86.54 & 9.24 & 850 & 60 & 5 & 1.1 & 0.9 & 0.100 & 0.108 \\
\hline J1456-6843 & 313.90 & -8.50 & 8.60 & 450 & 60 & 8 & 32.4 & 4.3 & 0.497 & 0.038 \\
\hline $\mathrm{J} 1932+1059$ & 47.38 & -3.88 & 3.18 & 330 & 10 & 5 & 18.5 & 0.9 & 0.269 & 0.036 \\
\hline J2018+2839 & 68.10 & -3.98 & 14.18 & 950 & 90 & 5 & 35.0 & 4.6 & 0.323 & 0.046 \\
\hline $\mathrm{J} 2022+2854$ & 68.86 & -4.67 & 24.64 & 2300 & 800 & 5 & 38.5 & 12.6 & 0.513 & 0.021 \\
\hline $\mathrm{J} 2022+5154$ & 87.86 & 8.38 & 22.65 & 1900 & 250 & 5 & 12.6 & 5.6 & 0.247 & 0.048 \\
\hline J0922+0638 & 225.42 & 36.39 & 27.27 & 1210 & 90 & 7 & 7.3 & 5.7 & 0.209 & 0.108 \\
\hline $\mathrm{J} 1537+1155$ & 19.85 & 48.30 & 11.61 & 1080 & 150 & 1 & 2.7 & 2.2 & 0.192 & 0.056 \\
\hline $\mathrm{J} 1713+0747$ & 28.75 & 25.22 & 15.99 & 910 & 80 & 1 & 4.9 & 2.8 & 0.172 & 0.102 \\
\hline J1744-1134 & 14.79 & 9.18 & 3.14 & 470 & 95 & 1 & 21.4 & 3.2 & 1.005 & 0.007 \\
\hline J1909-3744 & 359.73 & -19.60 & 10.39 & 1140 & 50 & 1 & 8.1 & 4.5 & 0.431 & 0.021 \\
\hline J0659+1414 & 201.11 & 8.26 & 13.98 & 290 & 30 & 4 & 14.0 & 1.2 & 0.085 & 0.567 \\
\hline J2145-0750 & 47.78 & -42.08 & 9.00 & 500 & 200 & 9 & 1.7 & 0.9 & 0.098 & 0.184 \\
\hline $\mathrm{J} 1022+1001$ & 231.79 & 51.10 & 10.25 & 400 & 145 & 1 & 2.7 & 1.3 & 0.129 & 0.198 \\
\hline J1024-0719 & 251.70 & 40.52 & 6.48 & 520 & 270 & 1 & 2.3 & 1.2 & 0.183 & 0.068 \\
\hline J2124-3358 & 10.93 & -45.44 & 4.60 & 250 & 165 & 1 & 2.8 & 0.9 & 0.189 & 0.098 \\
\hline $\mathrm{J} 0030+0451$ & 113.14 & -57.61 & 4.33 & 300 & 90 & 2 & 1.0 & 0.4 & 0.099 & 0.146 \\
\hline $\mathrm{J} 1509+5531$ & 91.32 & 52.29 & 19.61 & 2370 & 220 & 3 & 1.0 & 1.0 & 0.049 & 0.169 \\
\hline J0534+2200 & 184.56 & -5.78 & 56.79 & 2000 & 75 & 11 & 23.3 & 8.1 & 0.143 & 0.198 \\
\hline $\mathrm{J} 1740-5340 \mathrm{c}$ & 338.20 & -11.90 & 71.80 & 2300 & 20 & 11 & 37.8 & 24.0 & 0.335 & 0.093 \\
\hline J0141+6009 & 129.10 & -2.10 & 34.80 & 2750 & 150 & 11 & 29.0 & 5.6 & 0.161 & 0.079 \\
\hline J1910-5959c & 336.50 & -25.60 & 33.68 & 4000 & 250 & 11 & 3.2 & 3.1 & 0.092 & 0.091 \\
\hline $\mathrm{J} 1824-2452 \mathrm{c}$ & 7.80 & -5.58 & 119.86 & 5700 & 750 & 11 & 76.6 & 53.1 & 0.443 & 0.047 \\
\hline $\mathrm{J} 1701-3006 \mathrm{c}$ & 353.60 & 7.30 & 114.56 & 6900 & 900 & 11 & 59.4 & 50.2 & 0.438 & 0.038 \\
\hline $\mathrm{J} 1911+0101 \mathrm{c}$ & 36.11 & -3.92 & 202.68 & 9500 & 800 & 12 & 39.2 & 29.3 & 0.145 & 0.147 \\
\hline $\mathrm{J} 1823-3021 \mathrm{c}$ & 2.79 & -7.91 & 86.84 & 8000 & 800 & 11 & 26.8 & 24.2 & 0.279 & 0.039 \\
\hline $\mathrm{J} 1804-0735 \mathrm{c}$ & 20.79 & 6.77 & 186.32 & 8400 & 1900 & 11 & 35.5 & 31.2 & 0.168 & 0.132 \\
\hline $\mathrm{J} 1748-2021 \mathrm{c}$ & 7.73 & 3.80 & 220.40 & 8400 & 2100 & 11 & 88.7 & 61.6 & 0.279 & 0.094 \\
\hline $\mathrm{J} 1721-1936 \mathrm{c}$ & 4.86 & 9.74 & 75.70 & 8600 & 1100 & 11 & 21.4 & 20.4 & 0.270 & 0.033 \\
\hline J0024-7204c & 305.92 & -44.89 & 24.60 & 4500 & 250 & 11 & 3.6 & $\cdots$ & $\ldots$ & $\ldots$ \\
\hline $\mathrm{J} 1518+0205 \mathrm{c}$ & 3.8 & 46.80 & 29.47 & 7500 & 400 & 11 & 2.1 & $\ldots$ & $\cdots$ & $\ldots$ \\
\hline $\mathrm{J} 1641+3627 \mathrm{c}$ & 59.00 & 40.91 & 30.36 & 7700 & 380 & 11 & 1.8 & $\ldots$ & $\ldots$ & $\ldots$ \\
\hline $\mathrm{J} 2129+1210 \mathrm{c}$ & 65.01 & -27.31 & 67.31 & 10300 & 650 & 11 & 5.0 & $\ldots$ & $\ldots$ & $\ldots$ \\
\hline
\end{tabular}

${ }^{a}$ Hobbs \& Manchester (2003): www . atnf.csiro.au/research/pulsar/psrcat.

${ }^{b}$ Mean of absolute values of positive and negative error.

${ }^{c}$ In globular cluster. The last four pulsars are at $|z|>3 \mathrm{kpc}$, and only used in the determination of the electron scale height (see Fig. 1).

References. (1) Hotan et al. (2006); (2) Lommen et al. (2006); (3) Chatterjee et al. (2005); (4) Brisken et al. (2003); (5) Brisken et al. (2002); (6) Gwinn et al. (1986); (7) Chatterjee et al. (2001); (8) Bailes et al. (1990); (9) Löhmer et al. (2004); (10) Stairs et al. (1998); (11) Gomez et al. (2001); (12) Heitsch \& Richtler (1999).

Similarly, the emission measure perpendicular to the Galactic plane is

$$
\begin{aligned}
E M_{\mathrm{p}} \sin |b|(z) & =\int_{0}^{\left|Z_{\mathrm{p}}\right|} n_{\mathrm{e}}^{2}(z) \mathrm{d} z=\int_{0}^{\left|Z_{\mathrm{p}}\right|} f(z) n_{\mathrm{c}}^{2}(z) \mathrm{d} z \\
& =f_{0} n_{\mathrm{c} 0}^{2} h\left(1-\exp \left(-2\left|Z_{\mathrm{p}}\right| / h\right)\right) / 2,
\end{aligned}
$$

where the scale height $h_{\mathrm{e}}$ in Eq. (5) is equal ${ }^{2}$ to $h / 2$.

We can derive the maximum of $D M \sin |b|$ and the scale height $h$ from the distribution of $D M \sin |b|(z)$ of our sample of 38 pulsars shown in Fig. 1. A two-parameter fit yielded $f_{0} n_{\mathrm{c} 0} h=21.7 \pm 1.5 \mathrm{~cm}^{-3} \mathrm{pc}$ and $h=0.93 \pm$ $0.13 \mathrm{kpc}$, giving $n_{\mathrm{e}}(0)=f_{0} n_{\mathrm{c} 0}=0.023 \pm 0.004 \mathrm{~cm}^{-3}$. As the maximum of $E M_{\mathrm{p}} \sin |b|$ equals $E M_{\mathrm{c}} \sin |b|$, we have $n_{\mathrm{e}}^{2}(0) h / 2=f_{0} n_{\mathrm{c} 0}^{2} h / 2=2.8 \pm 0.3 \mathrm{~cm}^{-6} \mathrm{pc}$ (see Sect. 2); hence

$\overline{2}$ Note that BMM defined $h$ as the scale height of $n_{\mathrm{e}}^{2}(z)$, thus their $h$ equals our $h_{\mathrm{e}}=h / 2$. $n_{\mathrm{e}}^{2}(0)=0.0060 \pm 0.0011 \mathrm{~cm}^{-6}, n_{\mathrm{c} 0}=0.26 \pm 0.03 \mathrm{~cm}^{-3}$ and $f_{0}=0.09 \pm 0.02$.

We then analysed the data in our sample using $h_{\mathrm{e}}=h / 2=$ $0.47 \mathrm{kpc}$ in Eq. (5), leaving out 4 pulsars in globular clusters at $|z|>3 \mathrm{kpc}$. As only a small part of their lines of sight passes through the electron layer, the mean electron densities along the line of sight are unrealistically low. Figure 2 shows the distribution of $E M_{\mathrm{p}} \sin |b|(z)$ for all pulsars. The steady increase of $E M_{\mathrm{p}} \sin |b|$ with $|z|$ agrees well with the expected variation (full line), but the scatter is larger than in Fig. 1.

\section{Results}

In this section we investigate the dependencies of $\left\langle n_{\mathrm{e}}\right\rangle,\left\langle n_{\mathrm{e}}^{2}\right\rangle, N_{\mathrm{c}}$ and $F_{\mathrm{v}}$ on $|z|$, and the relationship between $F_{\mathrm{v}}$ and $N_{\mathrm{c}}$. The statistical treatment is the same as used by BMM and we refer to that paper for details. The results are given in Table 2 . 
Table 2. Statistical relationships derived for the sample of 34 pulsars.

\begin{tabular}{lllcccr}
\hline \hline$X$ & $Y$ & Fit & $a$ & $b$ & $\begin{array}{c}\text { Corr. } \\
\text { coeff. }\end{array}$ & $\begin{array}{c}\text { Student } \\
t^{6}\end{array}$ \\
\hline$D M$ & $E M_{\mathrm{p}}$ & bis $^{1}$ & $0.13_{-0.03}^{+0.04}$ & $1.15 \pm 0.07$ & $0.90 \pm 0.08$ & 11.8 \\
$D M \cos |b|$ & $E M_{\mathrm{p}} \cos |b|$ & $\prime$ & $0.13_{-0.02}^{+0.03}$ & $1.15 \pm 0.05$ & $0.93 \pm 0.06$ & 14.7 \\
$D M \sin |b|$ & $E M_{\mathrm{p}} \sin |b|$ & $\prime$ & $0.21 \pm 0.04$ & $0.98 \pm 0.09$ & $0.82 \pm 0.10$ & 8.3 \\
$D$ & $\left\langle n_{\mathrm{e}}\right\rangle$ & $\operatorname{lin}^{2}$ & $0.018 \pm 0.002$ & $-(2 \pm 6) 10^{-4}$ & $0.52 \pm 0.15$ & 3.5 \\
$|z|$ & $\left\langle n_{\mathrm{e}}\right\rangle$ & $\exp ^{3}$ & $0.019 \pm 0.002$ & $-3_{-3}^{+1}$ & $0.72 \pm 0.12$ & 5.9 \\
$|z|$ & $\left\langle n_{\mathrm{e}}^{2}\right\rangle$ & $\prime \prime$ & $0.0045 \pm 0.0007$ & $-1.4_{-0.6}^{+0.3}$ & $0.77 \pm 0.11$ & 6.9 \\
$|z|$ & $N_{\mathrm{c}}$ & $\prime \prime$ & $0.24_{-0.03}^{+0.04}$ & $-2.6_{-4.1}^{+1.0}$ & $0.76 \pm 0.12$ & 6.6 \\
$|z|$ & $F_{\mathrm{v}}$ & $\prime$ & $0.077_{-0.016}^{+0.020}$ & {$[+23]^{5}$} & $0.63 \pm 0.14$ & 4.6 \\
$|z|$ & $L_{\mathrm{e}} \sin |b|$ & pow $^{4}$ & $80 \pm 20$ & $1.06 \pm 0.15$ & $0.83 \pm 0.10$ & 8.5 \\
$N_{\mathrm{c}}$ & $F_{\mathrm{v}}$ & $\prime \prime$ & $0.011_{-0.002}^{+0.003}$ & $-1.20 \pm 0.13$ & $0.88 \pm 0.09$ & 10.2 \\
$N_{\mathrm{c}}$ & $L_{\mathrm{e}}$ & $\prime \prime$ & $24_{-11}^{+20}$ & $-0.85 \pm 0.35$ & $0.75 \pm 0.12$ & 6.5 \\
$N_{\mathrm{c}}$ & $L_{\mathrm{e}} \sin |b|$ & $\prime$ & $2 \pm 1$ & $-1.6 \pm 0.3$ & $0.82 \pm 0.10$ & 8.0 \\
\hline
\end{tabular}

Units: $D M$ in $\mathrm{cm}^{-3} \mathrm{pc}, E M$ and $E M_{\mathrm{p}}$ in $\mathrm{cm}^{-6} \mathrm{pc},\left\langle n_{\mathrm{e}}\right\rangle$ and $N_{\mathrm{c}}$ in $\mathrm{cm}^{-3},\left\langle n_{\mathrm{e}}^{2}\right\rangle$ in $\mathrm{cm}^{-6},|z|$ in $\mathrm{kpc}, L_{\mathrm{e}}$ in pc.

1) Bisector fit to powerlaw $Y=a X^{b}$ in $\log X-\log Y$ plane.

2) Linear fit; regression line of $Y$ on $X$, where $Y=a+b X$.

3) Exponential fit; regression line of $\ln Y$ on $|z|$ with slope $1 / b$, where $b$ is the scale height $H$ in kpc.

4) Powerlaw fit; regression line of $\log Y$ on $\log X$.

5) Undetermined: errors $>1 / b$.

6) Student test: for a sample of 34 pulsars the correlation is significant at the $3 \sigma$ level if $t>3.3$.

All fits are ordinary least-squares fits with errors of one standard deviation.

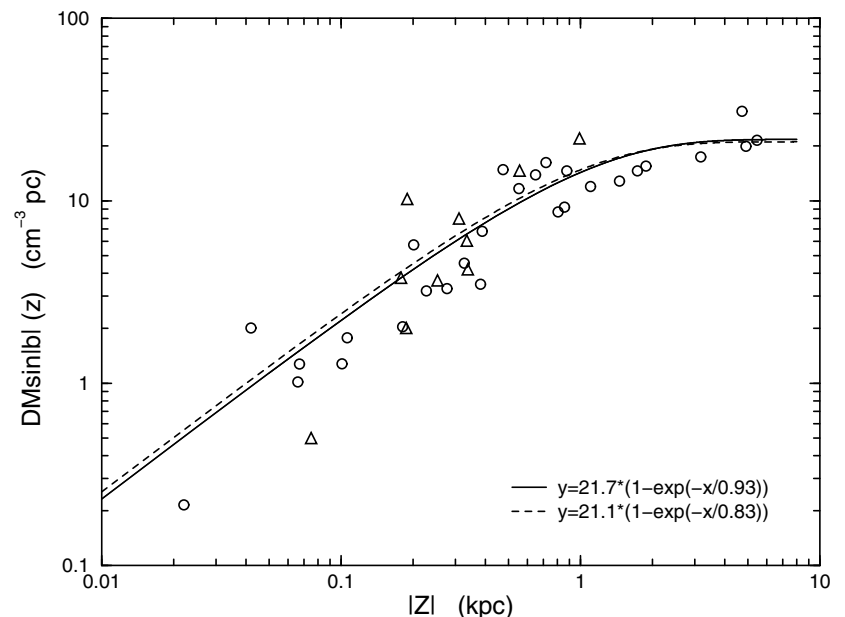

Fig. 1. Dependence of the dispersion measure perpendicular to the Galactic plane, $D M \sin |b|$, on distance to the plane, $|z|$, of the final sample of 38 pulsars. Circles: pulsars with $\Delta D / D<0.2$; triangles: pulsars with $0.2<\Delta D / D<0.5$. Full line: the two-parameter fit to the data; dashed line: fit to the original sample of 78 pulsars, shown for comparison.

We first show in Fig. 3 that $E M_{\mathrm{p}}$ is well correlated with $D M$ : the bisector fit yields $E M_{\mathrm{p}}=(0.13 \pm 0.04) D M^{1.15 \pm 0.07}$ with very high significance. This indicates that both quantities probe the same ionized regions along the line of sight, although the beams used for the $\mathrm{H} \alpha$ observations (up to $1^{\circ}$ ) also sample regions located around the single sightline to the pulsar. Variations in electron density across the beam will contribute to the scatter in Fig. 3. The components of $E M_{\mathrm{p}}$ and $D M$ along $|z|$ correlate less well than those along the Galactic plane (see Table 2), because of the different scale heights of $n_{\mathrm{e}}^{2}(z)$ and $n_{\mathrm{e}}(z)$.

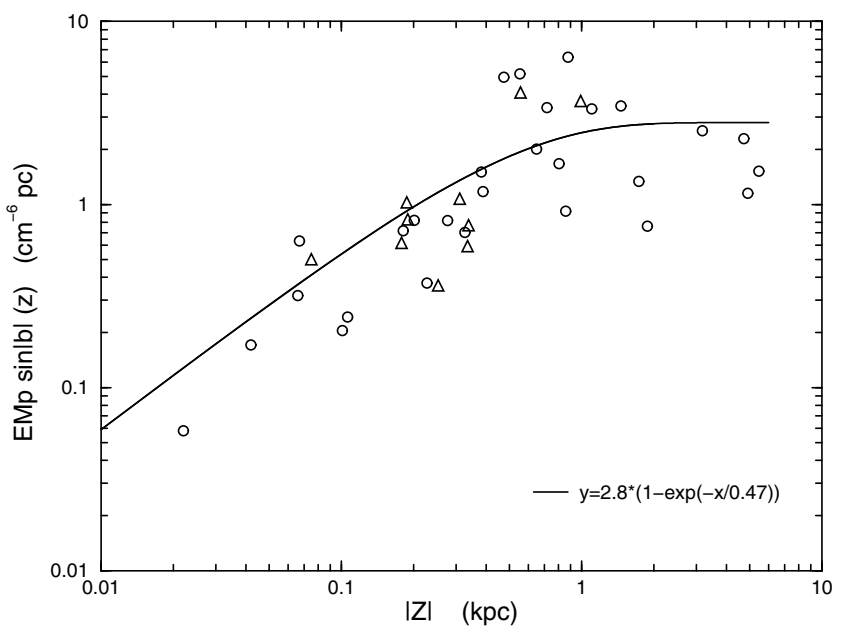

Fig. 2. Dependence of the corrected emission measure perpendicular to the Galactic plane, $E M_{\mathrm{p}} \sin |b|$, on distance to the plane, $|z|$, of the final sample of 38 pulsars. Circles: pulsars with $\Delta D / D<0.2$; triangles: pulsars with $0.2<\Delta D / D<0.5$. Full line: dependence expected for the scale height of $0.47 \mathrm{kpc}$ used in Eq. (5).

\subsection{Dependence of $\left\langle n_{\mathrm{e}}\right\rangle,\left\langle n_{\mathrm{e}}^{2}\right\rangle, N_{\mathrm{c}}$ and $F_{\mathrm{v}}$ on height}

Figure 4 presents the various densities and $F_{\mathrm{v}}$ as a function of $|z|$ in the $\log Y-|z|$ plane. Although these variables represent averages along the line of sight, we approximated their $|z|-$ distributions by exponentials with scale height $H$. The fits are listed in Table 2.

The distribution of $\left\langle n_{\mathrm{e}}\right\rangle(z)$ in Fig. 4a is effectively constant up to $|z| \simeq 1 \mathrm{kpc}$ (dashed line), a well known fact first noted by Weisberg et al. (1980). Beyond this height $\left\langle n_{\mathrm{e}}\right\rangle$ decreases. A fit to all points (full line) gives a mean value at the midplane of $\left\langle n_{\mathrm{e}}\right\rangle_{0}=0.019 \pm 0.02 \mathrm{~cm}^{-3}$, in fair agreement with the expected value $\left\langle n_{\mathrm{e}}\right\rangle_{0}=n_{\mathrm{e}}(0)=0.023 \pm 0.004 \mathrm{~cm}^{-3}$ derived in Sect. 3 . The 


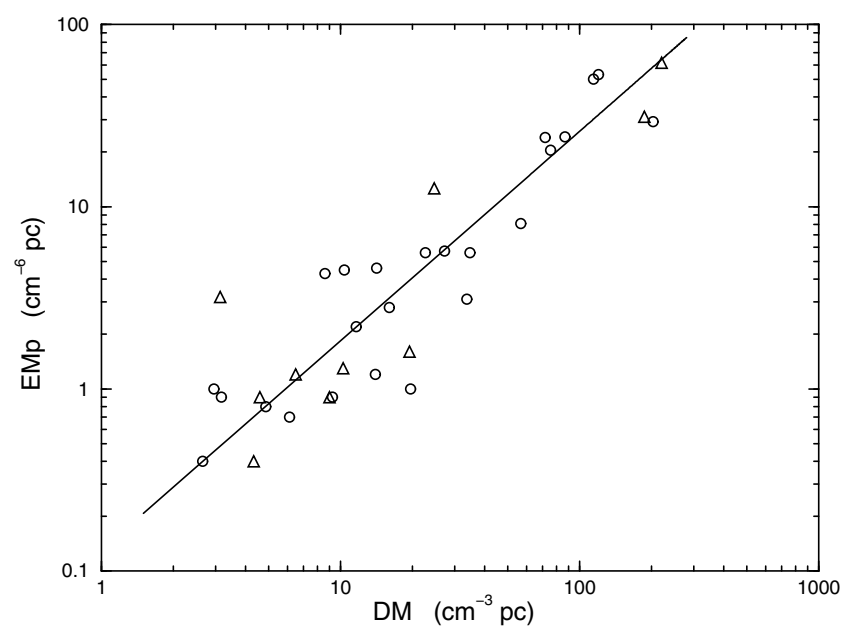

Fig. 3. Comparison of corrected emission measure, $E M_{\mathrm{p}}$, and observed dispersion measure, $D M$. Circles: pulsars with $\Delta D / D<0.2$; triangles: pulsars with $0.2<\Delta D / D<0.5$. Full line: bisector fit given in Table 2 .
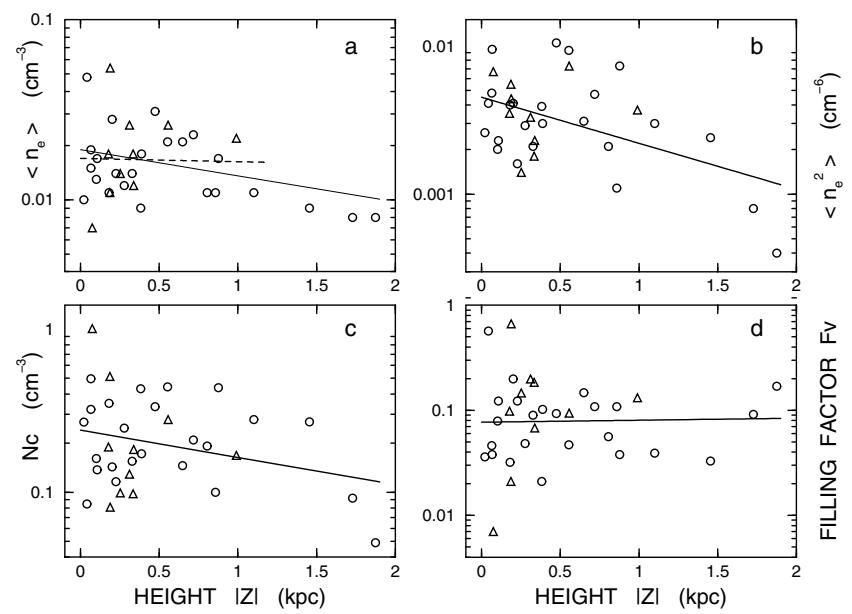

Fig. 4. Electron densities averaged along the line of sight and average volume filling factor as a function of height above the Galactic plane, $|z|$. a) Average density $\left\langle n_{\mathrm{e}}\right\rangle=D M \sin |b| /|z|$. The dashed line is the exponential fit to the data at $|z|<1.2 \mathrm{kpc}$. b) Average of the square of the density $\left\langle n_{\mathrm{e}}^{2}\right\rangle=E M_{\mathrm{p}} \sin |b| /|z|$. c) Mean density in clouds $N_{\mathrm{c}}=E M_{\mathrm{p}} / D M$. d) Average volume filling factor $F_{\mathrm{v}}=D M^{2} /\left(E M_{\mathrm{p}} D\right)$. Full lines indicate the exponential fits given in Table 2. Circles: pulsars with $\Delta D / D<0.2$; triangles: pulsars with $0.2<\Delta D / D<0.5$.

scale height is less well determined, though. The spread in the data clearly decreases away from the plane, as was also noted by BMM. This is not due to longer pathlengths, because the spread in the distribution projected along the plane remains constant (not shown). It just shows that the variety in electron density is larger near the Galactic plane than away from the plane.

The other panels in Fig. 4 show the dependencies of $\left\langle n_{\mathrm{e}}^{2}\right\rangle$, $N_{\mathrm{c}}$ and $F_{\mathrm{v}}$ on $|z|$. All quantities vary considerably about the fitted lines. The mean density in clouds slowly decreases from $0.24 \mathrm{~cm}^{-3}$ at $|z|=0 \mathrm{kpc}$ to $0.16 \mathrm{~cm}^{-3}$ at $|z|=1 \mathrm{kpc}$, but at all heights values between $0.08 \mathrm{~cm}^{-3}$ and $0.5 \mathrm{~cm}^{-3}$ occur. The midplane values of the exponential fits in Table 2 agree to within errors with those of $n_{\mathrm{e}}^{2}(0), n_{\mathrm{c} 0}$ and $f_{0}$ derived in Sect. 3 and the scale height $H$ of $\left\langle n_{\mathrm{e}}^{2}\right\rangle$ is indeed about half that of $\left\langle n_{\mathrm{e}}\right\rangle$ and $N_{\mathrm{c}}$. This agreement shows that the data are internally consistent.

The slight increase in $F_{\mathrm{v}}(z)$ in Fig. 4 d is statisically insignificant, thus $F_{\mathrm{v}}(z)$ is effectively a constant of about $0.08 \pm 0.02$. This differs from the clear increase in $F_{\mathrm{v}}(z)$ found by BMM for

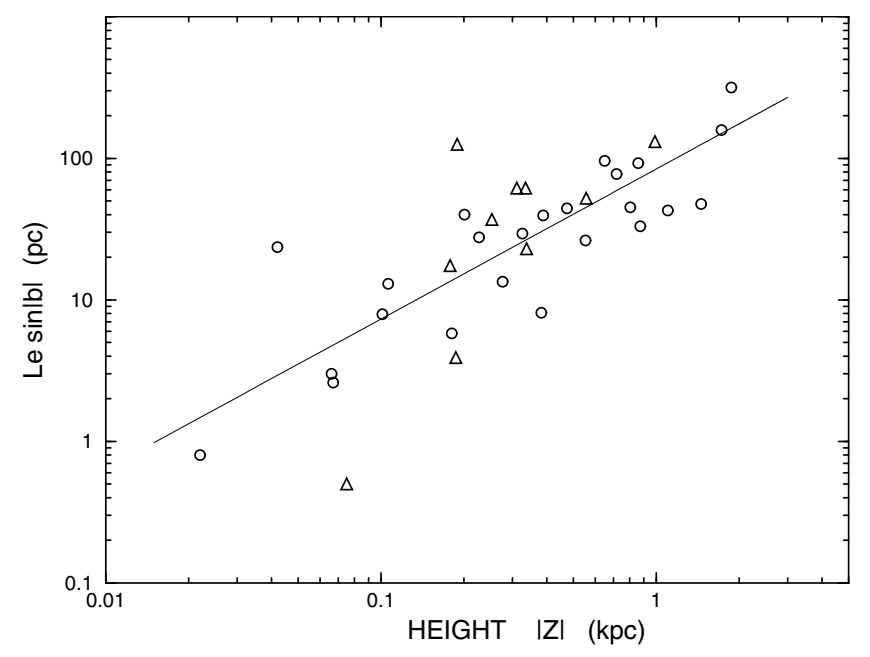

Fig. 5. Dependence of the total pathlength through ionized regions perpendicular to the Galactic plane, $L_{\mathrm{e}} \sin |b|$, on height above the plane. Circles: pulsars with $\Delta D / D<0.2$; triangles: pulsars with $0.2<$ $\Delta D / D<0.5$. Full line: powerlaw fit given in Table 2.

a much larger sample of pulsars. The spread in $F_{\mathrm{v}}$ seems largest near the Galactic plane, but this impression is mainly due to three extreme points of nearby pulsars at low $|z|$ : two near $F_{\mathrm{v}}=0.6$ and one near $F_{\mathrm{v}}=0.07$. At $|z|>0.3 \mathrm{kpc}$ the spread stays within a factor of 4 .

If the mean filling fraction remains about constant along the line of sight, the total pathlength through the ionized regions will increase linearly with distance. $L_{\mathrm{e}} \sin |b|(z)$ indeed increases nearly linearly from about $7 \mathrm{pc}$ towards $|z|=0.1 \mathrm{kpc}$ to about 80 pc towards $|z|=1 \mathrm{kpc}$ (see Fig. 5 and Table 2).

\subsection{Dependence of $F_{\mathrm{v}}$ on $N_{\mathrm{c}}$}

One of the indications for turbulence in the ISM is an inverse relationship between filling factor and mean cloud density. This is not only expected on theoretical grounds (Fleck 1996; Elmegreen 1999), but has also been obtained from simulations (Elmegreen 1997; Kowal \& Lazarian 2007). Observational evidence, however, is scarce (see Sect. 1).

Since $\left\langle n_{\mathrm{e}}\right\rangle$ is about constant (Fig. 4a) and $\left\langle n_{\mathrm{e}}\right\rangle=F_{\mathrm{v}} N_{\mathrm{c}}$, we expect an inverse relationship between $F_{\mathrm{v}}$ and $N_{\mathrm{c}}$. We present this relationship for our sample in Fig. 6. The correlation between $F_{\mathrm{v}}$ and $N_{\mathrm{c}}$ is very good and indeed nearly inverse: the power-law fit yields $F_{\mathrm{v}}\left(N_{\mathrm{c}}\right)=(0.011 \pm 0.003) N_{\mathrm{c}}^{-1.20 \pm 0.13}$ with a correlation coefficient of $0.88 \pm 0.09$, and covers about 1.5 decade in $N_{\mathrm{c}}\left(0.05-1 \mathrm{~cm}^{-3}\right)$ and $F_{\mathrm{v}}(0.4-0.01)$. We compare this result with earlier determinations and discuss its meaning for the density structure in Sect. 5.3.

As $F_{\mathrm{v}}\left(N_{\mathrm{c}}\right)$ is well defined, we looked for variations with longitude, distance along the plane and height above the plane that may indicate variations in structure in the DIG. We only found a dependence on height: at $|z|<0.3 \mathrm{kpc}$ the relationship is considerably steeper and the filling factor for $N_{\mathrm{c}}=1 \mathrm{~cm}^{-3}$ considerably smaller than at $|z|>0.3 \mathrm{kpc}$ (see Fig. 7 and Table 3 ). The two lines cover nearly the same range in $N_{\mathrm{c}}$ and cross near $N_{\mathrm{c}}=0.15 \mathrm{~cm}^{-3}$, but at $|z|>0.3 \mathrm{kpc}$ the spread in the data is larger than at lower $|z|$. On examination of the much larger sample of BMM, it appears to show the same trend (see Table 3). The large number of pulsars even permits a division of the lower $|z|-$ interval yielding exponents of $-1.6 \pm 0.2$ for $|z|<0.2 \mathrm{kpc}$ and $-1.1 \pm 0.1$ for $0.2<|z|<0.3 \mathrm{kpc}$. It seems that in the 
Table 3. $|z|$-dependence of the relation $F_{\mathrm{v}}\left(N_{\mathrm{c}}\right)=a N_{\mathrm{c}}^{b}$.

\begin{tabular}{lcccccc}
\hline \hline Sample & $\begin{array}{c}|z| \\
{[\mathrm{kpc}]}\end{array}$ & \multicolumn{1}{c}{$a$} & $b$ & $N$ & $\begin{array}{c}\text { Corr. } \\
\text { coeff. }\end{array}$ & $\begin{array}{c}\text { Student } \\
t^{1}\end{array}$ \\
\hline This & $0.0-0.3$ & $0.0070 \pm 0.0018$ & $-1.55 \pm 0.15$ & 15 & $0.94 \pm 0.09$ & 10.2 \\
work & $0.3-2.0$ & $0.021 \pm 0.006$ & $-0.80 \pm 0.17$ & 19 & $0.80 \pm 0.14$ & 5.6 \\
BMM & $0.0-0.2$ & $0.0078 \pm 0.0020$ & $-1.62 \pm 0.16$ & 11 & $0.97 \pm 0.08$ & 12.7 \\
& $0.2-0.3$ & $0.0183 \pm 0.0037$ & $-1.11 \pm 0.12$ & 24 & $0.91 \pm 0.09$ & 10.1 \\
& $0.0-0.3$ & $0.0137 \pm 0.0023$ & $-1.29 \pm 0.10$ & 35 & $0.92 \pm 0.07$ & 13.9 \\
& $0.3-2.0$ & $0.0187 \pm 0.0014$ & $-1.04 \pm 0.03$ & 122 & $0.95 \pm 0.03$ & 31.9 \\
\hline
\end{tabular}

1) Student test: for a sample of $N \geq 11$ pulsars the correlation is significant at the $3 \sigma$ level if $t>4.0$.

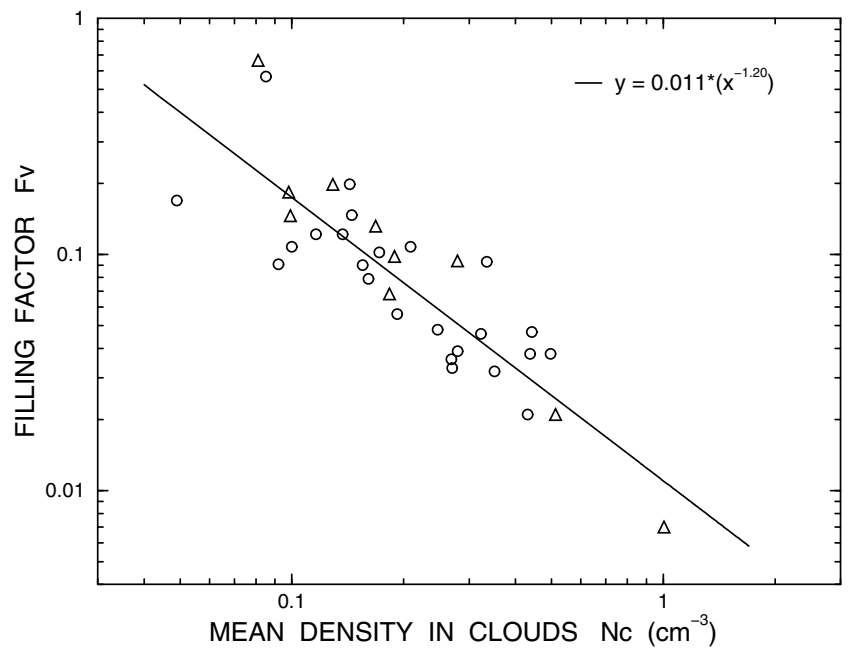

Fig. 6. Dependence of the average volume filling factor, $F_{\mathrm{v}}$, on the mean density in clouds, $N_{\mathrm{c}}$, for the sample of 34 pulsars in the solar neighbourhood. Circles: pulsars with $\Delta D / D<0.2$; triangles: pulsars with $0.2<\Delta D / D<0.5$. The full line shows the powerlaw fit given in Table 2.

thin Galactic disc $F_{\mathrm{v}}$ depends more strongly on $N_{\mathrm{c}}$ than above $|z|=0.2 \mathrm{kpc}$, with an exponent significantly smaller than -1 . This indicates that the structure of the DIG in the thin disc differs from that away from the plane. This may not be surprising. In the thin disc the activities of stellar winds, classical H II regions and supernova remnants largely determine the structure of the DIG. They may change or destroy the structure of the turbulence that is typical of more quiet regions away from the Galactic plane. Interestingly, Cordes \& Lazio (2002) derived a higher fluctuation factor for the thin disc than for the thick disc in their model of the electron density distribution. There is also evidence from measurements of interstellar scintillation that different types of turbulent spectra exist in different regions of the Galaxy (Shishov et al. 2003).

\section{Discussion}

\subsection{Comparison with BMM 2006}

It is interesting to compare our results with those of BMM who took the distances to the pulsars from the NE2001 model of Cordes \& Lazio (2002) and used an average correction for the absorption of the $\mathrm{H} \alpha$ emission that only depends on latitude. Applying their method to our sample, we recalculated the main relationships using a scale height of $0.47 \mathrm{kpc}$ to correct for $\mathrm{H} \alpha$ emission from behind the pulsar. Table 4 shows that the resulting relations are nearly identical to those derived in Sect. 4 , but

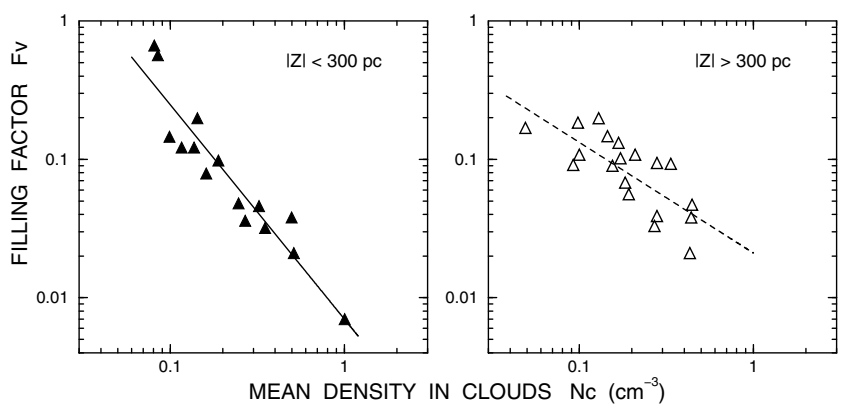

Fig. 7. Dependence of the relation $F_{\mathrm{v}}\left(N_{\mathrm{c}}\right)$ on distance to the Galactic plane $|z|$. Left: $|z|<0.3 \mathrm{kpc}$; right: $|z|>0.3 \mathrm{kpc}$. The lines are powerlaw fits given in Table 3 .

with larger errors. This means that the less accurate distances and absorption corrections used by BMM have not influenced their statistical results. Therefore, we may compare results from our small sample with those from the much larger sample analysed by BMM with confidence.

A striking difference between the two samples is the behaviour of $F_{\mathrm{v}}(z)$. Where BMM found a significant increase of the filling factor to about 0.2 towards $|z|=1 \mathrm{kpc}, F_{\mathrm{v}}(z) \mathrm{de}-$ rived from our sample remains constant within the errors at a value of about 0.08 . This difference must be due to the different locations in the Galaxy of the pulsars in the two samples. For example, 17 out of the 34 pulsars $(50 \%)$ in our sample are within $1 \mathrm{kpc}$ from the Sun, whereas only 44 out of 157 pulsars (28\%) of the BMM sample are within this distance. Since the ISM is a highly variable medium, differences between samples are to be expected. Hydromagnetic simulations of the evolution of the ISM by de Avillez \& Breitschwerdt (2005) may illustrate this point. Their Fig. 2a shows the density variation in a cut of $1 \mathrm{kpc}$ along and $10 \mathrm{kpc}$ perpendicular to the Galactic plane. Although the filling fraction of the DIG generally increases with height, there are also regions where it remains constant or even decreases away from the plane.

\subsection{Extent of ionized regions}

The total pathlength through ionized regions perpendicular to the Galactic plane increases nearly linearly from about $7 \mathrm{pc}$ towards $|z|=100 \mathrm{pc}$ to about $40 \mathrm{pc}$ towards $|z|=500 \mathrm{pc}$ and about $80 \mathrm{pc}$ towards $|z|=1 \mathrm{kpc}$ (Fig. 5).

In the ISM dense regions are usually smaller than less dense regions, which also applies to the DIG (see Fig. 8). Although the distribution of $L_{\mathrm{e}} \sin |b|$ with $N_{\mathrm{c}}$ shows considerable spread, it is well fitted by a power law (see Table 2). Regions of density $N_{\mathrm{c}}=1 \mathrm{~cm}^{-3}$ together occupy about $2 \mathrm{pc}$ in the $|z|$-direction and those of $N_{\mathrm{c}}=0.05 \mathrm{~cm}^{-3}$ about $300 \mathrm{pc}$. 
Table 4. Comparison with BMM for our sample of 34 pulsars.

\begin{tabular}{ccccc}
\hline \hline Relation & \multicolumn{2}{c}{ Our data } & \multicolumn{2}{c}{ BMM } \\
& $a$ & $b$ & $a$ & $b$ \\
\hline$\left\langle n_{\mathrm{e}}^{2}\right\rangle=a \mathrm{e}^{-|z| / b}$ & $0.0045 \pm 0.0007$ & $1.4_{-0.3}^{+0.6}$ & $0.0053 \pm 0.0011$ & $1.4_{-0.5}^{+1.5}$ \\
$N_{\mathrm{c}}(z)=a \mathrm{e}^{-|z| / b}$ & $0.24 \pm 0.04$ & $2.6_{1.0}^{+4.1}$ & $0.26 \pm 0.06$ & $2_{-1}^{+10}$ \\
$F_{\mathrm{v}}(z)=a \mathrm{e}^{|z| / b}$ & $0.077 \pm 0.018$ & {$[23]^{1}$} & $0.077 \pm 0.023$ & {$[5]^{1}$} \\
$F_{\mathrm{v}}\left(N_{\mathrm{c}}\right)=a N_{\mathrm{c}}^{b}$ & $0.011 \pm 0.003$ & $-1.20 \pm 0.13$ & $0.014 \pm 0.003$ & $-1.18 \pm 0.11$ \\
\hline
\end{tabular}

1) Undetermined: $1 / b$ smaller than errors.

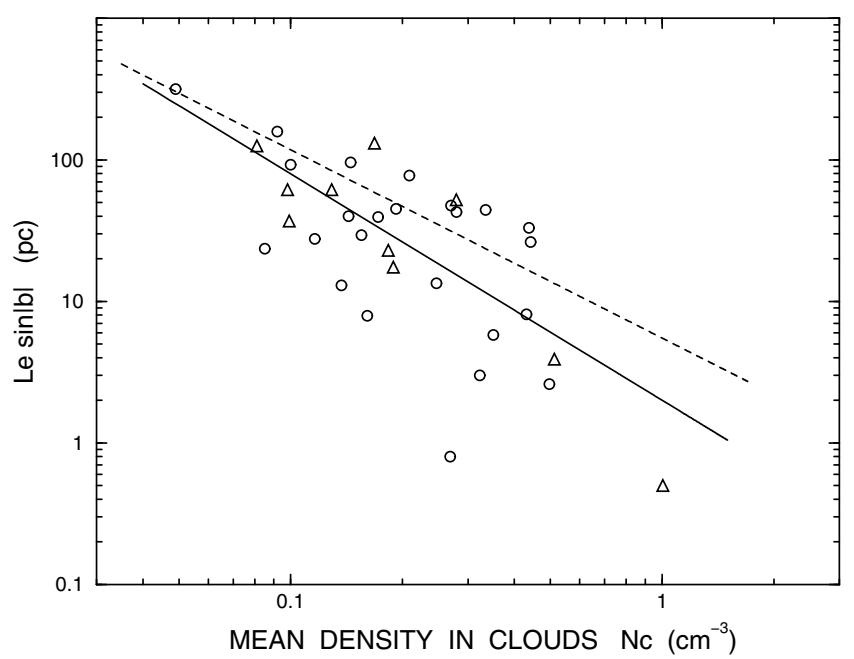

Fig. 8. Dependence of the total pathlength through ionized regions perpendicular to the Galactic plane, $L_{\mathrm{e}} \sin |b|$, on the mean density in clouds, $N_{\mathrm{c}}$. Circles: pulsars with $\Delta D / D<0.2$; triangles: pulsars with $0.2<\Delta D / D<0.5$. Full line: powerlaw fit given in Table 2; dashed line: powerlaw fit to the sample of 157 pulsars of BMM, $L_{\mathrm{e}} \sin |b|\left(N_{\mathrm{c}}\right)=$ $(5.5 \pm 0.8) N_{\mathrm{c}}^{-1.32 \pm 0.07}$.

We calculated the same relationship for the BMM sample which gives a somewhat larger extent for the higher densities (see Fig. 8). This power law is close to the one indicated by the distribution of 192 pulsars presented by Hill et al. (2007). However, these authors did not apply any correction to the observed emission measures, which leads to a flattening of the distribution and a shift to higher values of $N_{\mathrm{c}}$ for the same pathlength (see Fig. 5 in BMM).

\subsection{The $F_{\mathrm{v}}-N_{\mathrm{c}}$ relation and density structure}

of our small sample of pulsars, the nearly inverse correlation between $F_{\mathrm{v}}$ and $N_{\mathrm{c}}$ shown in Fig. 6 is very tight, reaching a correlation coefficient of $0.88 \pm 0.09$ (see Table 2). It does not change with distance along the plane and, as BMM explained, is insensitive to errors in emission measure. The near constancy of $\left\langle n_{\mathrm{e}}\right\rangle=F_{\mathrm{v}} N_{\mathrm{c}}$ along the line of sight (see $D-\left\langle n_{\mathrm{e}}\right\rangle$ fit in Table 2), first noted by Weisberg et al. (1980), was an early indication of an inverse relationship between $F_{\mathrm{v}}$ and $N_{\mathrm{c}}$.

We first compare our $F_{\mathrm{v}}-N_{\mathrm{c}}$ relation with earlier determinations available in the literature, and then discuss what it tells us about the density structure in the DIG.

\subsubsection{Comparison with other data}

Figure 9 and Table 5 show our $F_{\mathrm{v}}-N_{\mathrm{c}}$ relation together with earlier determinations. For the same mean density in clouds, we find

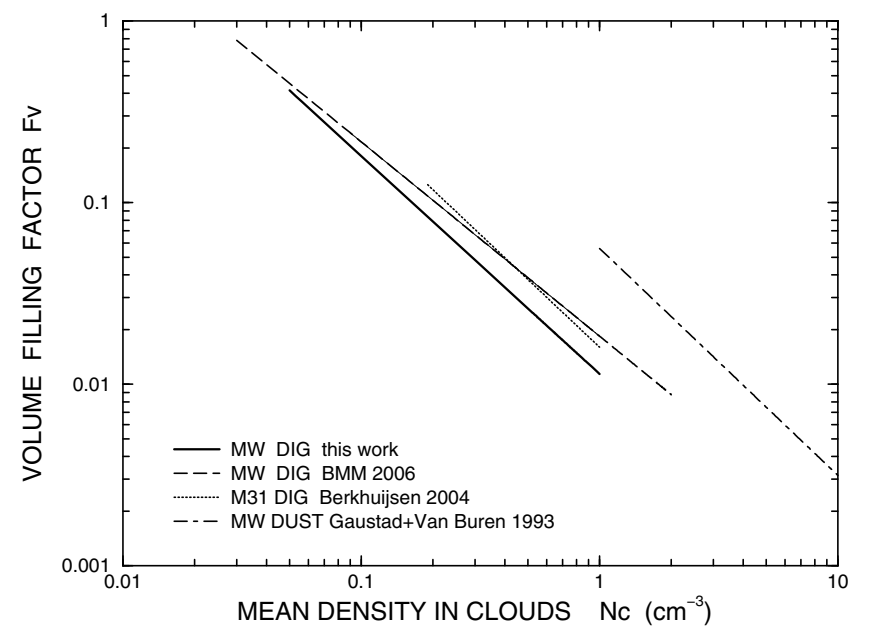

Fig. 9. Dependence of the volume filling factor, $F_{\mathrm{v}}$, on mean density in clouds, $N_{\mathrm{c}}$, as derived by different authors. Full line: this work; dashed line: dependence for the DIG within about $3 \mathrm{kpc}$ from the Sun (BMM); dotted line: dependence for the DIG in the bright emission ring in M31 (Berkhuijsen 2004); dash-dot line: dependence for clouds of diffuse dust within 400 pc from the Sun (Gaustad \& Van Buren 1993). The parameters of the lines are given in Table 5.

somewhat smaller filling factors than BMM which agree with the lower points in their distribution. The smaller errors in the BMM relation are not only due to the larger sample, but also to the smooth distribution of $\left\langle n_{\mathrm{e}}\right\rangle=D M / D$ of the electron density model of Cordes \& Lazio (2002) used by BMM. Berkhuijsen (2004) obtained the $F_{\mathrm{v}}-N_{\mathrm{c}}$ relation for the DIG in the galaxy M31 from a comparison of rotation measures of the polarized continuum emission (giving $\left\langle n_{\mathrm{e}}\right\rangle$ with magnetic field strengths of Fletcher et al. 2004) and thermal radio emission (giving $\left\langle n_{\mathrm{e}}^{2}\right\rangle$ ). These data refer to the DIG in the bright emission ring in M 31. The good agreement between the M 31 and the MW results suggests that the inverse correlation between $F_{\mathrm{v}}$ and $N_{\mathrm{c}}$ is generally valid in the DIG in galaxies.

Roshi \& Anantharamaiah (2001) observed radio recombination lines from the inner Galaxy at low latitudes. They derived filling factors of $\leq 0.01$ for extended regions of diffuse ionized gas with densities of $1-10 \mathrm{~cm}^{-3}$, which agree well with the extension of the curves for the DIG in Fig. 9.

Thus the inverse correlation between $F_{\mathrm{v}}$ and $N_{\mathrm{c}}$ at least holds for the density range $0.03-10 \mathrm{~cm}^{-3}$. Cordes et al. (1985) estimated a filling factor of $10^{-4.0 \pm 0.3}$ for clumps of about $1 \mathrm{pc}$ size causing scattering of pulsar signals. Our data predict this filling factor for clumps of density $N_{\mathrm{c}}=50 \mathrm{~cm}^{-3}$ and size $L_{\mathrm{e}} / m=0.9 \mathrm{pc}$, where $m$ is the number of clumps on the line of sight. The relation of BMM gives clumps of $N_{\mathrm{c}}=100 \mathrm{~cm}^{-3}$ for the same filling factor and sizes. So these clump properties also seem to follow the $F_{\mathrm{v}}-N_{\mathrm{c}}$ relations derived for the DIG. 
Table 5. Comparison of $F_{\mathrm{v}}-N_{\mathrm{c}}$ relations $F_{\mathrm{v}}\left(N_{\mathrm{c}}\right)=a N_{\mathrm{c}}^{b}$.

\begin{tabular}{|c|c|c|c|c|c|}
\hline Sample & $a$ & $b$ & $\begin{array}{c}\text { Range } N_{\mathrm{c}} \\
{\left[\mathrm{cm}^{-3}\right]}\end{array}$ & Range $F_{\mathrm{v}}$ & Ref. \\
\hline DIG & & & & & \\
\hline$N=34$ & $0.0114 \pm 0.0025$ & $-1.20 \pm 0.13$ & $0.05-1$ & $0.4-0.011$ & 1 \\
\hline MW $\quad N=157$ & $0.0184 \pm 0.0011$ & $-1.07 \pm 0.03$ & $0.03-2$ & $0.8-0.009$ & 2 \\
\hline M31 & $0.016 \pm 0.004$ & $-1.24 \pm 0.30$ & $0.19-1$ & $0.13-0.016$ & 3 \\
\hline DIFFUSE DUST & & & & & \\
\hline$D<400 \mathrm{pc}$ & $0.056 \pm 0.020$ & $-1.25 \pm 0.40$ & $1-10$ & $0.06-0.003$ & 4 \\
\hline
\end{tabular}

References. (1) This work; (2) Berkhuijsen et al. (2006); (3) Berkhuijsen (2004); (4) Gaustad \& Van Buren (1993).

Furthermore, Gaustad \& Van Buren (1993) obtained a similar relationship for clouds of diffuse dust within $400 \mathrm{pc}$ from the Sun, with mean densities of $1-10 \mathrm{~cm}^{-3}$. Figure 9 shows that these clouds have about 3 times higher filling factors than ionized clouds of the same mean density. Interestingly, their data agree very well with those of Pynzar (1993; also shown in Berkhuijsen 1998) derived from recombination lines.

\subsubsection{Density structure of the DIG}

The general validity of the inverse $F_{\mathrm{v}}-N_{\mathrm{c}}$ relation indicates that it describes a basic property of the DIG, possibly even of the entire ISM (Berkhuijsen 1999). At least two mechanisms could be responsible: thermal pressure equilibrium and turbulence causing a fractal density structure.

Thermal pressure equilibrium leads to an inverse $F_{\mathrm{v}}-N_{\mathrm{c}}$ relation if it is widespread, but it seems only locally valid. In the MW large fractions of gas are observed in unstable regimes and have also been found in simulations of a turbulent ISM. Thermal pressure equilibrium of clouds appears to be of minor importance in the presence of turbulence (see Elmegreen \& Scalo 2004, and references therein).

Elmegreen $(1998,1999)$ discussed the properties of diffuse ionized gas in a pervasive fractal ISM. One of these properties is an inverse correlation between volume filling factor and gas density, which is expected on theoretical grounds (Fleck 1996) as well as from simulations (Elmegreen 1997; Kowal \& Lazarian 2007). As this leads to a constant average density along the line of sight, the observed near constancy of $\left\langle n_{\mathrm{e}}\right\rangle$ with $N_{\mathrm{c}}$ and $F_{\mathrm{v}}$ inversely varying over 1.5 decade may indicate that the density distribution of the DIG is fractal. However, the near constancy of $\left\langle n_{\mathrm{e}}\right\rangle$ alone should only be regarded as a first indication, because the fits in Table 2 show that the statistical significance of the near constancy of $\left\langle n_{\mathrm{e}}\right\rangle$ is much lower than that of the nearly inverse $F_{\mathrm{v}}-N_{\mathrm{c}}$ relation.

The fractal medium is characterized by filamentary, clumpy structures that tend to cluster together, with holes inside and large voids between them. The filling factor along the line of sight is determined by the outer regions of the filaments, i.e. by the largest scales, because the small, dense clumps in the inner parts hardly contribute. The voids occupy more space than the filaments, thus the filling factor of the filaments is small. The values of $F_{\mathrm{v}} \simeq 0.1$ (Sect. 4.1) and $F_{\mathrm{v}} \simeq 0.2$ (BMM) obtained when looking perpendicular to the Galactic plane towards $|z|=1 \mathrm{kpc}$ are consistent with this picture.

The tendency of filaments to cluster into larger complexes reduces the number of fractal clouds, $N_{\mathrm{f}}$, along the line of sight. Elmegreen (1998) estimated $N_{\mathrm{f}}=3 / \mathrm{kpc}$ locally. For $F_{\mathrm{v}}=0.1$ the total pathlength through the ionized regions is $100 \mathrm{pc} / \mathrm{kpc}$, giving a line of sight through one fractal complex of about $30 \mathrm{pc}$ and a mean density $N_{\mathrm{c}}$ of $0.15 \mathrm{~cm}^{-3}$ (from Fig. 6). Using the results of BMM gives $F_{\mathrm{v}}=0.2$, a fractal complex of about $70 \mathrm{pc}$ along the line of sight and $N_{\mathrm{c}}=0.10 \mathrm{~cm}^{-3}$. Interestingly, these pathlengths are in the range of $10-100$ pc that Ohno \& Shibata (1993) estimated for the cells of ionized gas causing $D M$ and rotation measure $R M$ towards pairs of pulsars close on the sky.

According to Elmegreen (1998), a pervasive fractal structure only develops in regions outside the influence of SN shells, sites of star formation, chimneys etc. that are concentrated near the Galactic plane. This may explain our result of Sect. 4.2 that the exponent of the $F_{\mathrm{v}}-N_{\mathrm{c}}$ relation is significantly smaller in the thin disc $(|z|<300 \mathrm{pc})$ than further from the plane where it is close to -1 (see Table 3 and Fig. 7).

We conclude that our results on the mean electron densities and volume filling factors of the DIG outside the thin disc are consistent with a fractal ionized medium caused by turbulence, while the structure in the thin disc is dominated by the effects of SN shocks, stellar winds, star formation and other forces. The difference in density structure between these two regimes could be further analysed when more pulsars with measured distance become available.

Recently, further observational evidence for a turbulent structure of the DIG and of the diffuse atomic gas has been presented. Hill et al. (2008) found that the probability distribution function (PDF) of the emission measures observed perpendicular to the Galactic plane at $|b|>10^{\circ}$ is lognormal, as is expected for a turbulent medium from MHD simulations of the ISM (Elmegreen \& Scalo 2004). Furthermore, Berkhuijsen \& Fletcher (2008) showed that the PDFs of the quantities $\left\langle n_{\mathrm{e}}\right\rangle,\left\langle n_{\mathrm{e}}^{2}\right\rangle$, $N_{\mathrm{c}}$ and $F_{\mathrm{v}}$ derived above as well as that of the mean H I density along the line of sight towards stars, $\left\langle n_{\mathrm{HI}}\right\rangle$, are lognormal, consistent with a turbulent origin of density structure in the diffuse gas.

\section{Summary}

We have used pulsar dispersion measures (Manchester et al. 2005) and extinction-corrected emission measures (Finkbeiner 2003; Dickinson et al. 2003) towards 38 pulsars with known distances for a statistical study of several parameters of the diffuse ionized gas (DIG) in the Solar neighbourhood. The emission measures were also corrected for contributions from beyond the pulsar distance. To avoid regions with strong absorption ( $>1 \mathrm{mag}$ ) and contributions from H II regions most pulsars in the sample are at Galactic latitudes $|b|>5^{\circ}$. The statistical results are collected in Tables 2 and 3.

Our main conclusions are:

1. From the scaling of dispersion measures with distance perpendicular to the Galactic plane, we find a scale height of the ionized layer of $0.93 \pm 0.13 \mathrm{kpc}$ and an electron density 
at the midplane of $0.023 \pm 0.004 \mathrm{~cm}^{-3}$, in good agreement with earlier determinations.

2. The dispersion measure $D M$ and the corrected emission measure $E M_{\mathrm{p}}$ are well correlated, indicating that they probe the same ionized regions. We may then combine them to derive the average densities along the line of sight, $\left\langle n_{\mathrm{e}}\right\rangle$ and $\left\langle n_{\mathrm{e}}^{2}\right\rangle$, the mean electron density in clouds in the line of sight, $N_{\mathrm{c}}$, the volume filling factor of these clouds, $F_{\mathrm{v}}$, and the total pathlength through the ionized regions, $L_{\mathrm{e}}$.

3. The total extent of the ionized regions perpendicular to the Galactic plane increases linearly reaching about $80 \mathrm{pc}$ towards $|z|=1 \mathrm{kpc}$.

4. The filling factor $F_{\mathrm{v}}$ remains essentially constant with $|z|$ at a mean value of $0.08 \pm 0.02$. Whether $F_{\mathrm{v}}(z)$ is constant or systematically increases with $|z|$ depends on the regions in the Galaxy probed by the pulsar sample.

5. The average electron density $\left\langle n_{\mathrm{e}}\right\rangle$ is about constant with a spread of a factor $\$ 2$ about the mean value of $0.018 \pm$ $0.002 \mathrm{~cm}^{-3}$. Since $\left\langle n_{\mathrm{e}}\right\rangle=F_{\mathrm{v}} N_{\mathrm{c}}$, an inverse relationship between $F_{\mathrm{v}}$ and $N_{\mathrm{c}}$ is expected.

6. We derived the relation $F_{\mathrm{v}}\left(N_{\mathrm{c}}\right)=(0.011 \pm 0.003) N_{\mathrm{c}}^{-1.20 \pm 0.13}$, which covers about 1.5 decade in $N_{\mathrm{c}}\left(0.05-1 \mathrm{~cm}^{-3}\right)$ and $F_{\mathrm{v}}$ $(0.4-0.01)$ (see Fig. 6). This is in good agreement with earlier determinations of the $F_{\mathrm{v}}-N_{\mathrm{c}}$ relations for the DIG (see Fig. 9).

7. Near the Galactic plane the dependence of $F_{\mathrm{v}}$ on $N_{\mathrm{c}}$ is significantly stronger than away from the plane. This indicates that the thin disc has a different turbulent structure than regions away from the plane.

8. The inverse relationship between $F_{\mathrm{v}}$ and $N_{\mathrm{c}}$, and hence also the near constancy of $\left\langle n_{\mathrm{e}}\right\rangle$, are consistent with a fractal density distribution in the DIG caused by turbulence which dominates the structure outside the thin disc.

Acknowledgements. We thank Dr. Rainer Beck for careful reading of the manuscript and useful suggestions, and the referee for helpful comments leading to improvements in the manuscript.

\section{References}

Bailes, M., Manchester, R. N., Kesteven, M. J., et al. 1990, Nature, 343, 240 Bennett, C. L., Hill, R. S., Hinshaw, G., et al. 2003, ApJS, 148, 97

Berkhuijsen, E. M. 1998, in The Local Bubble and Beyond, ed. D. Breitschwerdt, M. J. Freyberg, \& J. Trümper, Lect. Notes Phys., 506, 301

Berkhuijsen, E. M. 1999, in Plasma Turbulence and Energetic Particles in Astrophysics, ed. M. Ostrowski, \& R. Schlickeiser (Krakow: Astron. Obs. Jagiellonian Univ.), 61

Berkhuijsen, E. M. 2004, Ap\&SS, 289, 207

Berkhuijsen, E. M., \& Fletcher, A. 2008, MNRAS Lett., in press [arXiv: 0806.4316V1]
Berkhuijsen, E. M., Mitra, D., \& Müller, P. 2006, AN, 327, 82 (=BMM) Bhattacharia, D., \& Verbunt, F. 1991, A\&A, 242, 128

Brisken, W. F., Benson, J. M., Goss, W. M., et al. 2002, ApJ, 571, 906

Brisken, W. F., Thorsett, S. E., Golden, A., et al. 2003, ApJ, 593, L89

Chatterjee, S., Cordes, J. M., Lazio, T. J. W., et al. 2001, ApJ, 550, 287

Chatterjee, S., Vlemmings, W. H. T., Brisken, T. J. W., et al. 2005, ApJ, 630, L61

Cordes, J. M., \& Lazio, T. W. J. 2002 [arXiv:astro-ph/0207156]

Cordes, J. M., \& Lazio, T. W. J. 2003 [arXiv: astro-ph/0301598]

Cordes, J. M., Weisberg, J. M., \& Boriakoff, W. 1985, ApJ, 288, 221

de Avillez, M. A., \& Breitschwerdt, D. 2005, A\&A, 436, 585

Dickinson, C., Davies, R. D., \& Davis, R. J. 2003, MNRAS, 341, 369

Diplas, A., \& Savage, B. D. 1994, ApJ, 427, 274

Elmegreen, B. G. 1997, ApJ, 477, 196

Elmegreen, B. G. 1998, PASAu, 15, 74

Elmegreen, B. G. 1999, in The Physics and Chemistry of the Interstellar Medium, 3rd Cologne-Zermatt Symposium, ed. V. Ossenkopf, J. Stutzki, \& G. Winnewisser (Aachen: Shaker), 77

Elmegreen, B. G., \& Scalo, J. 2004, ARA\&A, 42, 211

Finkbeiner, D. P. 2003, ApJ, 146, 407

Fleck, R. C. 1996, ApJ, 458, 739

Fletcher, A., Berkhuijsen, E. M., Beck, R., \& Shukurov, A. 2004, A\&A, 414, 53 Gaustad, J. E., \& Van Buren, D. 1993, PASP, 105, 1127

Gomez, G. C., Benjamin, R. A., \& Cox, D. P. 2001, AJ, 122, 908

Gwinn, C. R., Taylor, J. H., Weisberg, J. M., et al. 1986, AJ, 91, 3383

Haffner, L. M., Reynolds, R. J., \& Tufte, S. L. 1998, ApJ, 501, L83

Haffner, L. M., Reynolds, R. J., Madsen, G. J., et al. 2003, ApJS, 149, 405

Heitsch, F., \& Richtler, T. 1999, A\&A, 347, 455

Hill, A. S., Reynolds, R. J., Benjamin, R. A., \& Haffner, L. M. 2007, ASP Conf. Ser., 365, 250

Hill, A. S., Benjamin, R. A., Kowal, G., et al. 2008, ApJ, in press [arXiv: 0805.0155V2]

Hinshaw, G., Nolta, M. R., Bennett, R., et al. 2007, ApJS, 170, 288

Hobbs, G. B., \& Manchester, R. N. 2003,

http://www . atnf.csiro.au/research/pulsar/psrcat

Hobbs, G., Lorimer, D. R., Lyne, A. G., \& Kramer, M. 2005, MNRAS, 360, 974

Hotan, A. W., Bailes, M., Ord, S. M., et al. 2006, MNRAS, 369, 1502

Kowal, G., \& Lazarian, A. 2007, ApJ, 666, L69

Kulkarni, S. R., \& Heiles, C. 1988, in Galactic and Extragalactic Radio Astronomy, ed. G.A. Verschuur, \& K.I. Kellermann (New York: Springer), 95

Löhmer, O., Kramer, M., Driebe, T., et al. 2004, A\&A, 426, 631

Lommen, A. N., Kipphorn, R. A., Nice, D. J., et al. 2006, ApJ, 642, 1012

Lorimer, D. R. 2004, in Young Neutron Stars and Their Environments, ed. F. Camilo, \& B. M. Gaensler (San Francisco: ASP), IAU Symp., 218, 105

Madsen, G. J., Reynolds, R. J., \& Haffner, L. M. 2006, ApJ, 652, 401

Manchester, R. N., Hobbs, G. B., Teoh, A., \& Hobbs, M. 2005, AJ, 129, 1993

Nordgren, T. E., Cordes, J. M., \& Terzian, Y. 1992, AJ, 104, 1465

Ohno, H., \& Shibata, S. 1993, MNRAS, 262, 953

Pynzar, A. V. 1993, ARep, 37, 245

Reynolds, R. J. 1991a, ApJ, 372, L17

Reynolds, R. J. 1991b, in The Interstellar Disk-Halo Connection in Galaxies, ed. H. Bloemen (Dordrecht: Kluwer), IAU Symp., 144, 67

Reynolds, R. J. 1997, in The Physics of Galactic Haloes, ed. H. Lesch, R. J. Dettmar, U. Mebold, \& R. Schlickeiser (Berlin: Akademie), 57

Roshi, D. A., \& Anantharamaiah, K. R. 2001, ApJ, 557, 226

Shishov, V. I., Smirnova, T. V., Sieber, W., et al. 2003, A\&A, 404, 557

Stairs, I. H., Arzoumanian, Z., Camilo, F., et al. 1998, ApJ, 505, 352

Weisberg, J. M., Rankin, J., \& Boriakoff, V. 1980, A\&A, 88, 84

Yusifov, I., \& Küçük, I. 2004, A\&A, 422, 545 